\title{
Performance analysis of liver tumor classification using machine learning algorithms
}

\author{
Munipraveena Rela ${ }^{*}$, Suryakari Nagaraja $\operatorname{Rao}^{2}$ and Patil Ramana Reddy ${ }^{3}$ \\ Research Scholar, Department of ECE, Jawaharlal Nehru Technological University Ananthapuramu, Ananthapur \\ India $^{1}$ \\ Professor, Department of ECE, G. Pulla Reddy Engineering College (Autonomous), Kurnool, Andhra Pradesh, \\ India $^{2}$ \\ Professor, Department of ECE, JNTUA College of Engineering, Ananthapuramu, Andhra Pradesh, India ${ }^{3}$
}

Received: 24-August-2021; Revised: 16-January-2022; Accepted: 20-January-2022

(C)2022 Munipraveena Rela et al. This is an open access article distributed under the Creative Commons Attribution (CC BY) License, which permits unrestricted use, distribution, and reproduction in any medium, provided the original work is properly cited.

\begin{abstract}
Liver is most important organ in human body. Mainly there are two types of liver cancers- "liver abscess (LA), and hepatocellular carcinoma (HCC)". Computed tomography (CT) is used to identify these liver cancers. The shape of liver, tumor, and tumor location changes with patients, hence it is very difficult to classify liver tumors. Early detection of liver cancers such as LA and HCC are very essential to reduce the mortality rate. Medical image analysis techniques are used for identification of liver abnormalities. In this paper, different machine learning algorithms such as support vector machine (SVM), K-Nearest neighbour $(K N N)$, decision tree $(D T)$, ensemble, and naive Bayes (NB) are used to classify the tumor as LA, and HCC. The steps required for classification are "preprocessing, liver segmentation, feature extraction, and classification". 68 CT images are collected from different hospitals in Tirupati to train the model, and the models are validated using accuracy, specificity, sensitivity, Matthew correlation coefficient (MCC), and F1-score etc. From the performance analysis of different classifiers, it is observations that accuracy of SVM classifier is improved by 10\%, specificity is improved by $40 \%$, sensitivity is improved by $28 \%$, precision is improved by $66.67 \%$, MCC is improved by $8 \%$, F1-score is improved 4\%, and kappa is improved by $20.14 \%$ compare to KNN, whereas error is reduced by $33 \%$. SVM performance is also improved by $22.22 \%, 45.83 \%, 40 \%$, 62\%, 20\%, and $70 \%$ with respect to accuracy, precision, specificity, MCC, F1-score, and kappa compared to DT classifier whereas $50 \%$ reduction in error. We can conclude that SVM classifier gives better performance compare to all other classifiers in the study.
\end{abstract}

\section{Keywords}

Liver, Liver tumor, CT images, Medical image analysis, Segmentation, SVM, KNN.

\section{Introduction}

The biggest glandular biological structure in the human body is liver. It is involved in a variety of bodily functions, including metabolism, digestion, and detoxification [1]. The spleen, stomach, pancreas, gall bladder, and intestine are related to this organ through blood arteries. Hepatic disorders have major effects on the remaining organs as a result of liver functional diversity and vascular connectivity. The fifth most common cancer in males is liver cancer across the world [2]. Figure 1 gives the images of liver abnormalities and corresponding computed tomography (CT) images.

*Author for correspondence

143
Medical imaging is crucial for tumor identification, its diagnosis, surgical planning and interventional therapy [3]. Because of its high spatial resolution, high imaging speed, and low cost, CT is frequently used for diagnosis, and multiphase contrast-enhanced CT is also commonly suggested for the abdomen imaging. Multiphase can increase the characterization and detection of liver abnormalities [4]. Contrast-enhancement makes liver tumor segmentation much more difficult because the characteristics of a tumor might change depending on scan delay, contrast dose, clinical stage, and tumor variety. CT image segmentation of the liver tumors has got a lot of attention due to the poor contrast and imprecise boundaries [5]. 
Liver abscess (LA) is a fluid-filled pus pocket within the liver. Abdominal infection, contagion in the blood, endoscopy and infection of the bile draining tubes, and liver trauma are causes of LA [6]. Despite the low prevalence of LA, it is critical to recognize and treat these lesions as soon as possible because they have a high death rate [7]. Hepatocellular carcinoma (HCC) is the frequent sort of primary malignant growth and a main source of disease related demise throughout the world [8]. Cirrhosis is the main danger cause for the improvement of HCC. Hepatitis B and C are the symptoms of cirrhosis [9]. Figure 2 shows that the tumour's appearance changes dramatically with phases. Liver tissue appears on CT images at a similar intensity to surrounding organs. Depending on the tumor type, and the pathological stage, the tumor's profile may vary on CT images. Furthermore, liver cancers have no fixed location. These challenges must be taken into account when developing a liver and tumor segmentation algorithm,

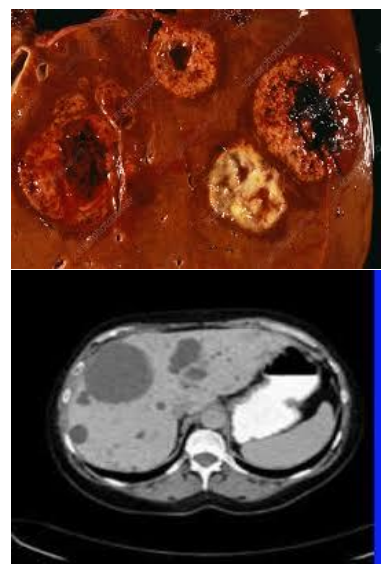

a
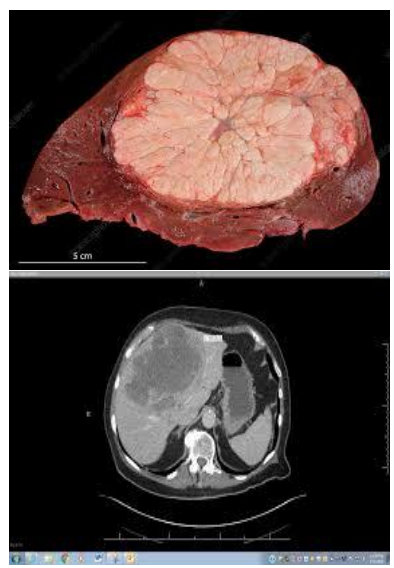

b it remains difficult in case of medical image processing [10].

The motivation is used to design a computer-aided design (CAD) systems for liver tumor identification and classification. There are huge number of liver tumor classification systems. These systems are not able to identify the tumor accurately. The objective of this paper is effective segmentation of liver, extraction of features from the CT images, and classification using machine learning and deep learning techniques.

The rest of this paper is structured as follows. The literature review is discussed in section 2. The methods for liver classification are explained in section 3, and feature extraction is addressed in section 4. The results are detailed in section 5. Discussion is included in section 6 and section 7 contains conclusion.
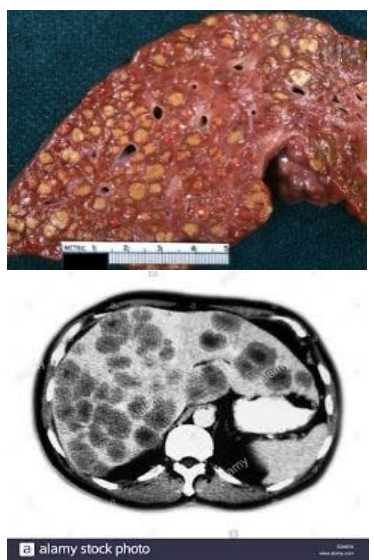

c
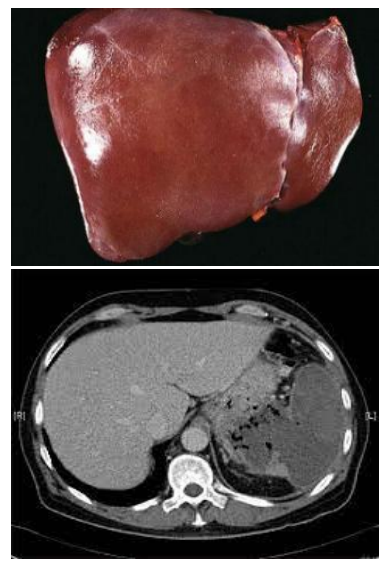

d

Figure 1 True Images and corresponding CT of (a). Liver abscess, (b). Hepatocellular carcinoma (c). Cirrhosis (d). Normal
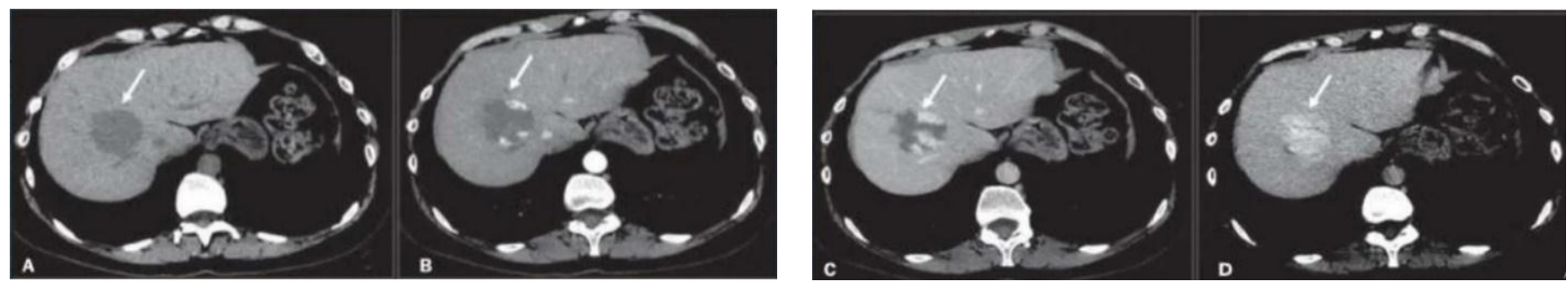

Figure 2 Phases of precontrast, arterial, portal venous, equilibrium

\section{Literature review}

Machhale et al. [11] proposed support vector machine (SVM) - K-nearest neighbour (KNN) hybrid classifier for brain tumor classification. In this paper, $\mathrm{KNN}$ is used to calculate nearest vectors to the hyperplane. They have shown that this method gives accuracy of $98 \%$.

Nadira and Rustam [12] used global artificial bee colony (GABC) - SVM method for breast cancer. It gives accuracy of $96.42 \%$. 
Starmans et al. [13] proposed radiomics approach for liver tumor classification. $119 \mathrm{~T} 2 \mathrm{w}$ magnetic resonance (MR) sequences with 424 features with hyper parameter optimisation are used for training and testing SVM model. Random-split crossvalidation is used to estimate the performance of the model.

Zhen et al. [14] used 31,608 images for training convolution neural network (CNN) to classify LTs as seven types, three types and binary. 6816 images are used to validate the model. The results show that seven-way classifier has poor performance.

Trivizakis et al. [15] proposed three dimensional (3D) CNN for classifying LTs from magnetic resonance imaging (MRI) images. They used rectified linear unit (ReLU) as activation function, 2048 neurons in fully connected layer, and soft max layer for classification. $130 \mathrm{MRI}$ images are used for training and validation. The results show that $3 \mathrm{D}$ CNN gives improved performance compare to two dimensional (2D) CNN.

Kutlu and Avc [16] developed a CNN-discrete wavelet transform (DWT) - long short-term memory (LSTM) method to classify LTs and brain tumors. In this paper, $\mathrm{CNN}$ is used for extraction $1 \times 4096$ feature, DWT is used to reduce feature vector to $1 \times 2054$ and LSTM is used for classification. The dataset consists of 112 LT images, and 300 brain tumor images. The results show that this method gives high accuracy compare to KNN and SVM classifiers.

Rela et al. [17] proposed two deep learning networks that is $\mathrm{CNN}$ and recurrent neural network (RNN) for LT region classification. The inputs for $\mathrm{CNN}$ are tumor segmented images and for RNN are features. Here opposition based spotted hyena optimization is used. This method gives improved accuracy compare to other heuristic based deep learning networks.

Balagourouchetty et al. [18] developed a model, which consist of two stage enhancement, lesion segmentation, modified GoogLeNet for deep feature extraction, and ensemble fully connected neural network (FCNet) classifier. This model is used to classify six types of liver CT images.

Budak et al. [19] used cascaded encoder-decoder CNNs for LT segmentation. First network is used for liver segmentation, this is given as input to second network, and the output of second network is tumor segmentation. These networks give improved segmentation accuracy compare to existing methods. Zhang et al. [20] developed a weakly-supervised teacher-student network for LT segmentation. In this network, teacher learns to segment from enhanced CT images, where student able to segment the unenhanced images.

Devi and Seenivasagam [21] discussed a CAD system based on SVM classifier using feature difference of lesion and liver. The dataset has $120 \mathrm{CT}$ images.

Krishan and Mittal [22] proposed ensemble of six classifiers for classification of HCC and metastases based on 44 features extracted from $1638 \mathrm{CT}$ images. This system accuracy is $100 \%$.

All the methods discussed in the literature classify the images as benign or malign. These methods are used to identify whether tumor is in early stage or final stage. If the tumor is in final stage, then survival of the patient is very difficult. If the tumor is in early stage, then survival rate of patient can be improved. The two malign tumors that cause most liver cancer deaths are LA and HCC. In this paper, we have collected 68 CT images of malign LTs from different hospitals across Tirupati, and classified these images as LA, and HCC. These two abnormalities are causing most liver cancer deaths worldwide. If the type of LT is identified, then particular medication can be used to cure the cancer. Here, in this paper, particular type of liver cancer can be identified.

\section{Methods}

In this paper, CT liver images are classified as LA, and HCC using different classification technique such as SVM, KNN, naive Bayes (NB), decision tree (DT), ensemble, and discriminate classifiers. The classifiers are trained using different features like local binary pattern (LBP), Gray-level co-occurrence matrix (GLCM), shape feature and intensity-based features. Performance of classifiers are studied using statistical parameters for classification. The block diagram in Figure 3 shows the implementation of this paper. Database consist of $68 \mathrm{CT}$ images with LA and HCC abnormalities. Liver region is segmented from $\mathrm{CT}$ images using region growing segmentation with manually selected seed point, then size of liver image is set to $256 \times 256$. 86 features extracted from liver region. Here, different machine learning models are trained using 80 percent of feature vectors to classify the images as LA and HCC. Then the models are tested using 20 percent of feature vectors. Finally, 
models are validated using different statistical parameters. From the results, it is observed that SVM gives good classification performance.
Different machine learning models used for classification are discussed in subsequent section.

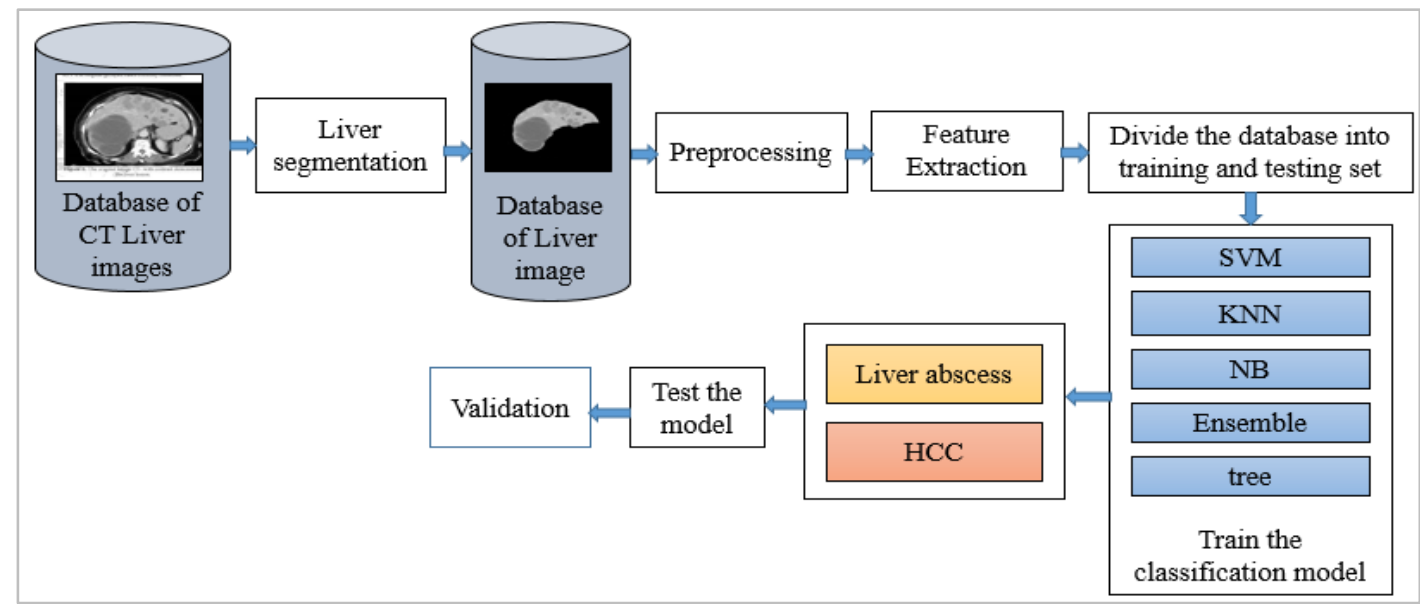

Figure 3 Block diagram representation of paper implementation

\subsection{SVM}

Let there are $l$ observations and each observation comprises of $\left\{\boldsymbol{i}_{\boldsymbol{x}}, j_{x}\right\}$, where $\boldsymbol{i}_{\boldsymbol{x}}$ is a vector, $\boldsymbol{i}_{\boldsymbol{x}} \in$ $R^{n}, x=1, \ldots, l$ and $j_{x}$ is the associated "class". $P(i, j)$ is probability distribution with which these observations are obtained. A machine has been designed with the objective of learning the mapping $\boldsymbol{i}_{\boldsymbol{x}} \rightarrow j_{x}$. The machine is described by a series of mappings $\boldsymbol{i} \rightarrow f(\boldsymbol{i}, \alpha)$, with the functions $f(\boldsymbol{i}, \alpha)$ being labelled by the changeable parameters $\alpha$. The machine is deterministic, which means it always produce the unchanged output $f(\boldsymbol{i}, \alpha)$ for the input $\boldsymbol{i}$, and option of $\alpha$. A certain selection of $\alpha$ generates a "trained machine." The trained machine has the test error as shown in Equation 1:

$$
R(\alpha)=\int \frac{1}{2}|j-f(i, \alpha)| d P(i, j)
$$

Where, $d P(\boldsymbol{i}, j)=p(\boldsymbol{i}, j) d \boldsymbol{i} d j$, and $p(\boldsymbol{i}, j)$ is the density function.

$R(\alpha)$ in equation 1 is the actual risk. The "empirical risk" $R_{e m p}(\alpha)$ is the mean error rate, given in Equation 2.

$R_{e m p}(\alpha)=\frac{1}{2 l} \sum_{x=1}^{l}\left|j_{x}-f\left(i_{x}, \alpha\right)\right|$

$R_{e m p}(\alpha)$ is a constant value for a certain value of $\alpha$ and for a specific $\left\{\boldsymbol{i}_{\boldsymbol{x}}, j_{x}\right\} \cdot \frac{1}{2}\left|\mathrm{j}_{\mathrm{x}}-\mathrm{f}\left(\mathrm{i}_{\mathrm{x}}, \alpha\right)\right|$ has two possible values: zero and one, and is referred to as the loss. Select $\eta$, its value is $0 \leq \eta \leq 1$. The following constrain in Equation 3 holds for losses with probability $1-\eta$ :

$R(\alpha) \leq R_{e m p}(\alpha)+\sqrt{\left(\frac{h\left(\log \left(\frac{2 l}{h}\right)+1\right)-\log \left(\frac{\eta}{4}\right)}{l}\right)}$

Where $h$ is the Vapnik Chervonenkis (VC) dimension, a positive integer that represents the understanding capacity. The VC dimension is a characteristic of a collection of $\{f(\alpha)\}$ that can be defined for a variety of function $f$.

In case of pattern recognition with two classes, $f(\boldsymbol{i}, \alpha) \in\{-1,1\} \forall \boldsymbol{i}, \alpha$. Suppose $\boldsymbol{i}_{\boldsymbol{x}} \in R^{2}$, and the $\{f(\alpha)\}$ comprises of directed straight lines, with all data on one side represent "class 1" and all data on another side, "class -1". An arrow in Figure 4 indicates the orientation, indicating which aspect of the line focuses ought to be the class 1 . The hyperplanes in $R^{n}$ has VC dimension of $n+1$, because one point is selected as origin in $n+1$ points, and the remaining $n$ points has linearly independent position vectors.

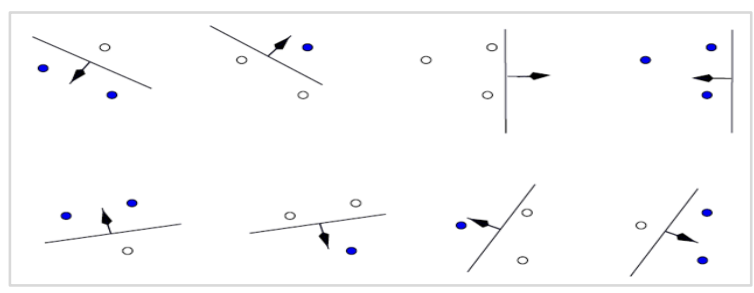

Figure 4 Points in $\mathrm{R}^{\wedge} 2$ 


\subsubsection{Linear SVM}

Let $\left\{i_{x}, j_{x}\right\}, x=1,2, \ldots, l$, are $l$ training data, $i_{x} \in$ $R^{d}, j_{x} \in\{-1,1\}$. A hyperplane separates the class 1 from the class -1 examples. The points $\boldsymbol{i}$ on the hyperplane fulfil $\boldsymbol{N} \cdot \boldsymbol{i}+b=0$, where $\boldsymbol{N}$ is the hyperplane's normal, $\|\boldsymbol{w}\|$ is the Euclidean norm of $\boldsymbol{N}$, and $|b| /\|\boldsymbol{N}\|$ is the hyperplane's normal distance from the origin. The smallest distance between the hyperplane and the closest negative (positive) is denoted by $d_{-}\left(d_{+}\right)$. A separating hyperplane's "margin" is defined as $d_{+}+d_{-}$. Assume that all of the training data meets the following criteria:

Equation 4 and Equation 5 are combined into Equation 6.

$i_{x} \cdot N+b \geq+1$ when $j_{x}=+1$

$i_{x} \cdot N+b \leq-1$ when $j_{x}=-1$

$j_{x}\left(i_{x} \cdot \boldsymbol{N}+b\right)-1 \geq 0, \forall x$

Consider $\mathrm{w}$ and $\mathrm{b}$ for which Equation 4 holds true, present on $H_{1}: i_{x} \cdot \boldsymbol{N}+b=1$ with perpendicular distance from the origin $|1-b| /\|N\|$, whereas, the points for which Equation 5 holds true, present on $H_{2}: i_{x} \cdot \boldsymbol{N}+b=-1$, with perpendicular distance from the origin $|-1-b| /\|\boldsymbol{N}\|$. Hence $d+=$ $d-=1 / \boldsymbol{N}$ with the margin $2 / \boldsymbol{N} . H_{1}$ and $H_{2}$ are hyperplanes with no data between them. Thus, by decreasing $\|\boldsymbol{N}\|^{2}$, we may identify the pair of hyperplanes that yields the biggest margin, subject to limitations Equation 6.

As a result, the solution for a $2 \mathrm{D}$ situation should take the form indicated in Figure 4. Support vectors are illustrated in Figure 5 by the extra circles. These are the training points for which Equation 6 holds, and removing them would modify the solution discovered;

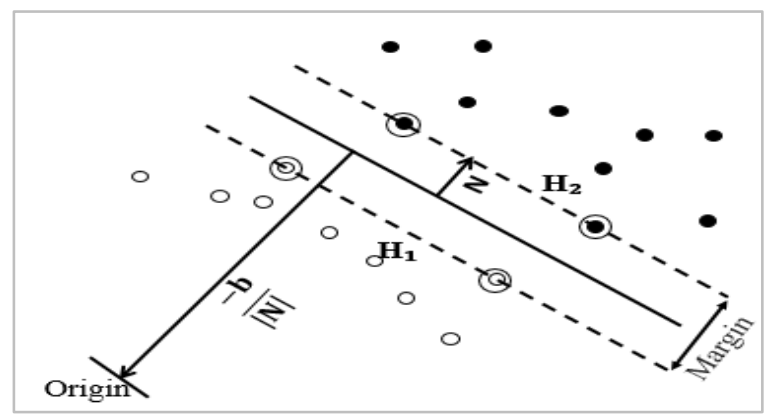

Figure 5 Hyperplanes with support vectors

Let us propose positive Lagrange multipliers $\alpha_{x}$, $x=1, \cdot \cdot, l$, , one each for limitations on disparity given in Equation 6. As a result, Lagrangian is as given Equation 7:

$L_{P} \equiv \frac{1}{2}\|\boldsymbol{N}\|^{2}-\sum_{x=1}^{l} \alpha_{x} j_{x}\left(i_{x} \cdot \boldsymbol{N}+b\right)+\sum_{x=1}^{l} \alpha_{x}$

The conditions are as follows when the gradient of $L_{P}$ with respect to $\boldsymbol{N}$ and b vanishes:

$\boldsymbol{N}=\sum_{i} \alpha_{i} y_{i} x_{i}$

$\sum_{i} \alpha_{i} y_{i}=0$

By substituting Equation 8, Equation 9 into Equation 7.

$L_{D}=\sum_{x} \alpha_{x}-\frac{1}{2} \sum_{x, y} \alpha_{x} \alpha_{y} j_{x} j_{y} i_{x} \cdot i_{y}$

$L_{P}$ is minimised to obtain the solution. The vectors for which $\alpha_{x}>0$ are called "support vectors" in the solution given by Equation 8 and lie on one of the $H_{1}, H_{2}$. All other training locations have $\alpha_{x}=0$ and are either on $H_{1}$ or $H_{2}$, or on the side of $H_{1}$ or $H_{2}$ such that Equation 6 holds.

\subsubsection{Nonlinear SVM}

Let the data is mapped to infinite dimensional Euclidean space $\mathcal{H}$, using a mapping $\psi$ in Equation 11:

$\psi: R^{d} \mapsto \mathcal{H}$

The training process would be dependent on the data via dot products in $\mathcal{H}$, i.e., on functions of the type $\psi\left(\mathrm{I}_{x}\right) \cdot \psi\left(\mathrm{I}_{y}\right)$.

Now, if there were a "kernel function" $K$ such that $K\left(\mathrm{i}_{\mathrm{x}}, \mathrm{i}_{\mathrm{y}}\right)=\psi\left(\mathrm{I}_{x}\right) \cdot \psi\left(\mathrm{I}_{y}\right)$. If $K\left(\mathrm{i}_{\mathrm{x}}, \mathrm{i}_{\mathrm{y}}\right)$ is substituted for $i_{x} \cdot i_{y}$, the training process will generate a SVM that operates in an unbounded dimensional space in roughly the same amount of time as training on unmapped data. The following were the kernels researched for the pattern recognition problem:

$K(i, j)=e^{-\|i-j\|^{2} / 2 \sigma^{2}}$

$K(i, j)=(i \cdot j+1)^{p}$

$K(i, j)=\tanh (\kappa i \cdot j-\delta)$

Equation 12 generates a Gaussian radial basis function; Equation 13 generates a polynomial of degree $p$ in the data classifier; and Equation 14 generates a specific type of two-layer sigmoidal neural network [23].

\subsection{The K-nearest neighbour (KNN) algorithms}

The labelled sample set $W=\left\{x_{1}, x_{2}, \ldots, x_{n}\right\}$ is does not need to be pre-processed before using the nearest neighbour classifiers. This technique allots an unknown-class input $\mathrm{y}$ to the nearest neighbour's class. The same rule can be applied to the KNN, 
where the unknown-class input y being allocated to the class that has the largest number of KNN [24].

\subsection{Naive bayes classification (NBC)}

The data is subjected to density estimate by NBC. Given the class, the algorithm uses Bayes theorem and asserts that the predictors are conditionally independent. NBC tends to provide posterior distributions that are robust to biased class density estimations, especially when the posterior is 0.5 (the decision boundary). The most likely class is assigned by NBC to observations

\subsection{Ensemble classification}

These are learning algorithms that build a group of classifiers and then categorise incoming data points based on a selection of their predictions. Ensembles have been discovered to be far more accurate than the individual classifiers that make them up. To solve the same problem, this learning multiple classifier system trains many hypotheses. Random forest trees are an example of ensemble modelling, in which a number of DTs are used to predict outcomes.

An ensemble as shown in Figure 6 is made up of several hypothesis or learners that are produced from training data using a basic learning method. Most ensemble methods produce homogeneous ensembles using a single base learning algorithm, whereas others build heterogeneous ensembles with numerous learning algorithms [25].

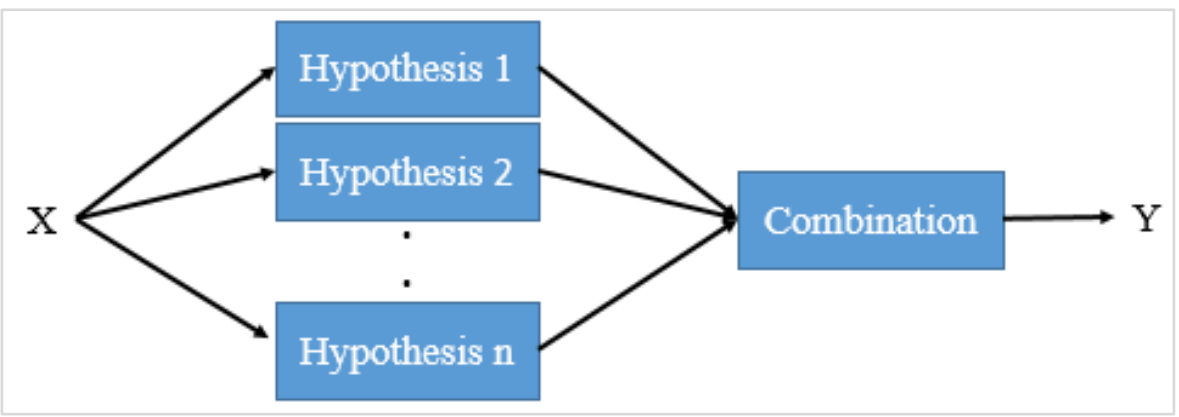

Figure 6 Architecture of ensemble

\section{Feature extraction}

\subsection{LBP}

LBP is a useful texture pattern descriptor for describing an image's local texture patterns. It's commonly utilized in image-processing-related applications [26]. The LBP operates on a $3 \times 3$ block size, with the centre pixel serving as a threshold for neighboring pixels, and the LBP code for a centre pixel being formed by encoding the determined threshold value.

LBP is given as in Equation 15 and Equation 16:
$L B P=\sum_{i=0}^{P-1} s\left(n_{i}-G_{c}\right) 2^{i}$
$s(x)= \begin{cases}1, & \text { if } x>0 \\ 0, & \text { otherwise }\end{cases}$

where $n_{i}$ is $i^{\text {th }}$ neighboring pixel, $\mathrm{P}$ denotes the number of neighboring pixels, and $c$ the centre pixel. The acquired LBP code is used to extract the histogram features of size $2^{P}$. As a result, the histogram feature vector length for eight nearby pixels is 256. With a $G_{c}$ value of 10 and eight neighbouring pixels, the LBP process is shown in Figure 7.

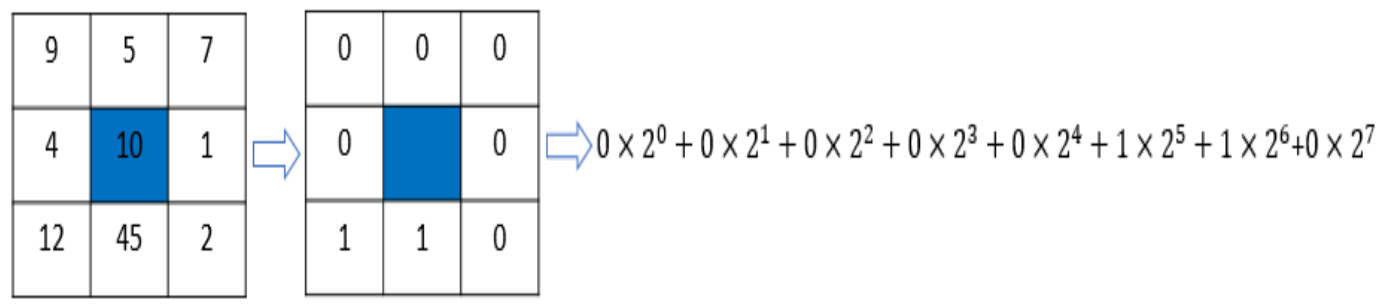

Figure 7 LBP operation 
4.2Gray level co-occurrence matrix (GLCM)

The GLCM is a technique for obtaining statistical texture information of second order. The GLCM is used to model the associations between pixels within the region. The GLCM is primarily depend on an assessment of 2nd-order joint conditional probability density $p(i, j \mid d, \theta)$ for various directions $d=$ $1,2,3,4$, and 5 and distancesd $=0,45,90,135^{\circ}$, etc. GLCM features "energy, contrast, correlation, and homogeneity" are given in Table1 [27].

Table 1 GLCM features

\begin{tabular}{lc}
\hline Energy & $\sum_{x, y=0}^{N-1}\left(Q_{x, y}\right)^{2}$ \\
\hline Contrast & $\sum_{x, y=0}^{N-1} Q_{x, y}(x-y)^{2}$ \\
\hline Correlation & $\sum_{x, y=0}^{N-1} Q_{x, y} \frac{(x-\mu)(y-\mu)}{\sigma^{2}}$ \\
\hline Homogeneity & $\sum_{x, y=0}^{N-1} \frac{Q_{x, y}}{1+(x-y)^{2}}$ \\
\hline Where & \\
Mean $=\mu=\sum_{x, y=0}^{N-1} x Q_{x, y}$ & \\
Variance $=\sigma^{2}=\sum_{x, y=0}^{N-1} Q_{x, y}(x-\mu)^{2}$
\end{tabular}

4.3Shape features

Initially image should be transformed to gray scale image, then obtained binary image by using imbinarize, then the following features are obtained from binary image: "number of connected components, area of binary image, perimeter, total number of pixels in perimeter, and centroid" using "bwlabel, bwarea, bwperim, sum(sum(bwperim)), and centroid" respectively.

\subsection{Intensity based features}

Features such as: "mean, standard deviation, entropy, root mean square (RMS), variance, smoothness, inverse difference movement, and skewness" are extracted using "mean2, std2, entropy, RMS, 1$(1 /(1+\quad \operatorname{sum}(\operatorname{double}(\mathrm{x}(:))))), \quad \sum_{i=1}^{m} \sum_{j=1}^{n} \mathrm{x}(\mathrm{i}, \mathrm{j}) /(1+$ $\left.(i-j)^{\wedge} 2\right)^{\prime \prime}$.

\section{Results}

The database consists of 86 features extracted from each liver image, there are 68 liver images labelled into two categories, LA, and HCC. The CT liver images collected from different hospitals in Tirupati. Each image is of size $800 \times 700.34 \mathrm{CT}$ liver images contain LA, and remaining 34 images contain HCC. These images are used to extract the features. The features data set size is $68 \times 86$ and $80: 20$ percentage of this data is used for training and testing. We have used different classifier like SVM, KNN, Ensemble, DT, NB and discriminant. Performance of these classifiers are measured using "accuracy, error, sensitivity, specificity, precision, false positive rate (FPR), F1-score, Matthews correlation coefficient (MCC), and kappa". Figure $8(a)$ is the plot of accuracy with respect to various classifiers. This plot says that SVM classifier gives $85 \%$ of correct predictions over total number of predictions whereas DT and ensemble classifier gives lowest accuracy for the dataset considered.

Figure $8(b)$ is the plot of error verses different classifiers. This plot shows that SVM classifier gives lowest number of incorrect classifications compare to other classifiers. Figure $8 \quad(c)$ is sensitivity measurement with respect to different classifiers, shows that discriminant analysis classifier has 100\% ability to predict LA as LA and HCC as HCC compare to other classifiers. Figure $8(d)$ is specificity variation with respect to different classifiers, shows that SVM classifiers gives $87.5 \%$ of not predictiong LA as HCC and HCC as LA. Figure $8(e)$ shows that SVM gives precision of $83.3 \%$, that not to label LA, if features belong to 
Munipraveena Rela et al.

HCC. Figure $8(f),(g),(h)$, and $(i)$ show that SVM gives lowest FPR of $12.5 \%$, that is incorrect prediction of liver abnormality, $80 \%$ of F1-score that is performance rate, $67.5 \%$ of MCC that is correctly

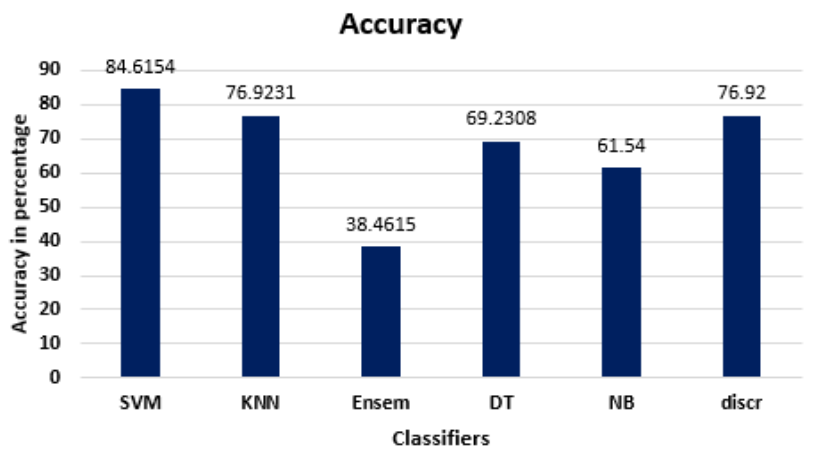

(a)

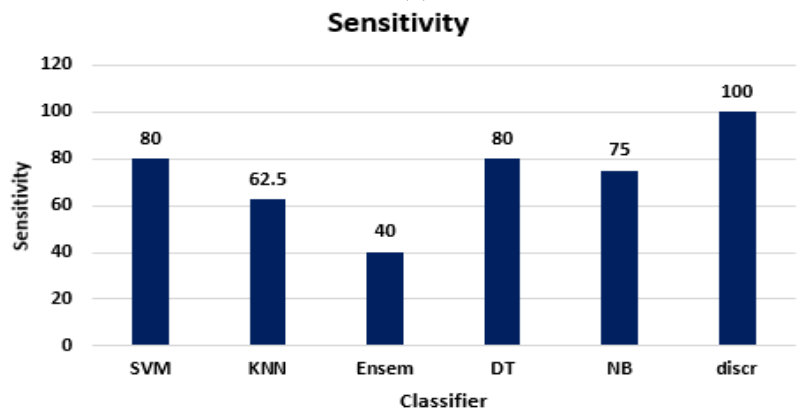

(c)

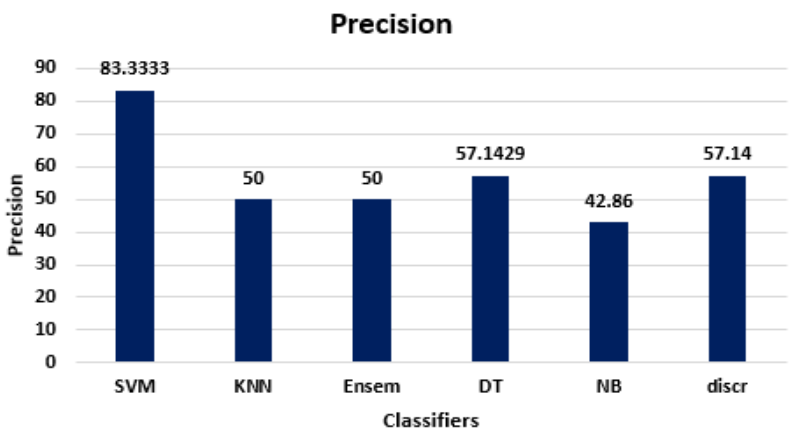

(e)

F1-score

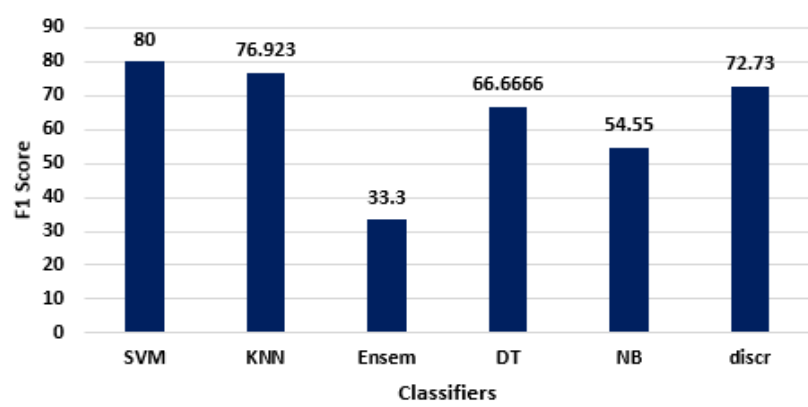

(g) predicting LA and HCC, and 67\% of Kappa. Overall performance of all the classifiers is shown in Figure 9.

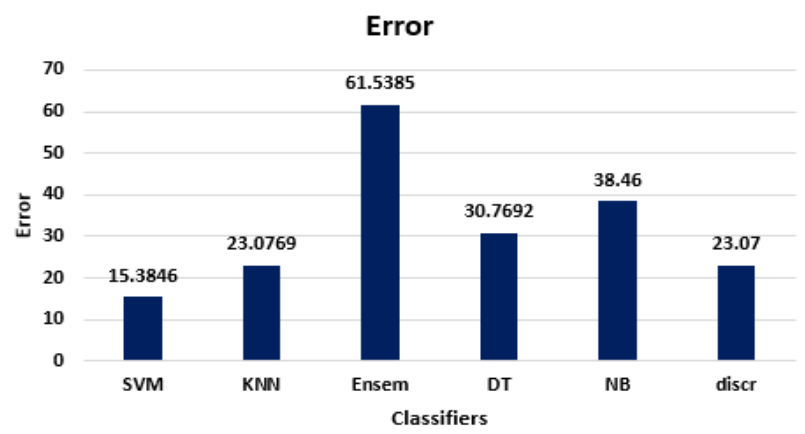

(b)

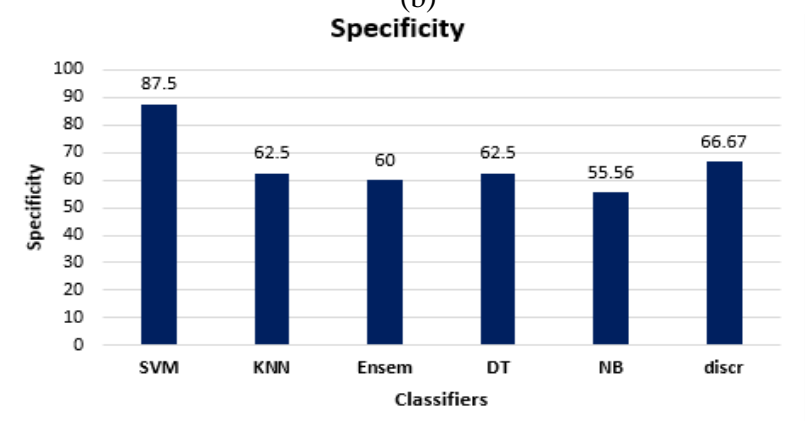

(d)

FPR

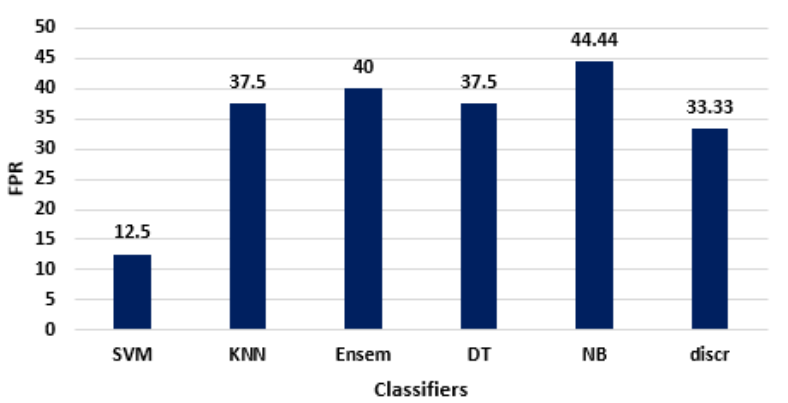

(f)

MCC

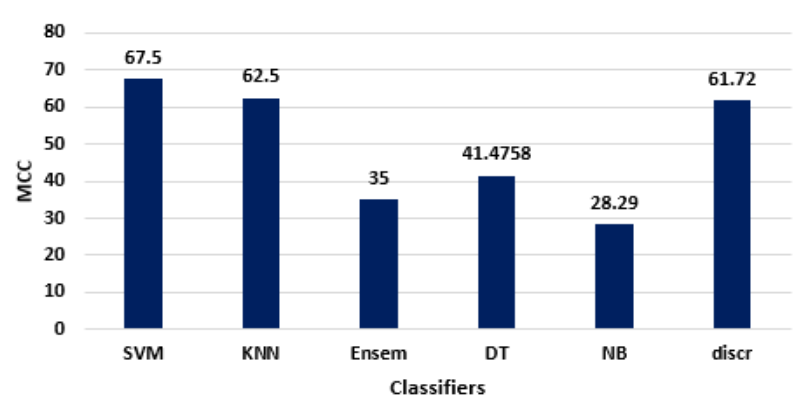

(h) 


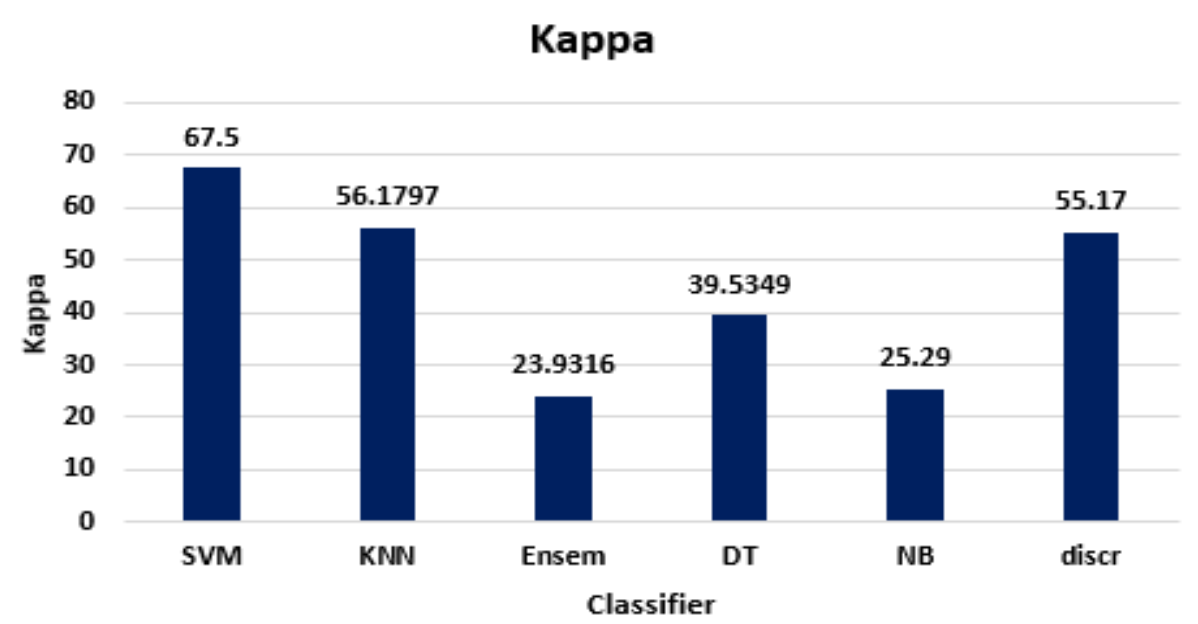

(i)

Figure 8 (a) Accuracy, (b) Error, (c) Sensitivity, (d) Specificity, (e) Precision, (f) FPR, (g) F1-score, (h) MCC, and (i) Kappa for different classifiers

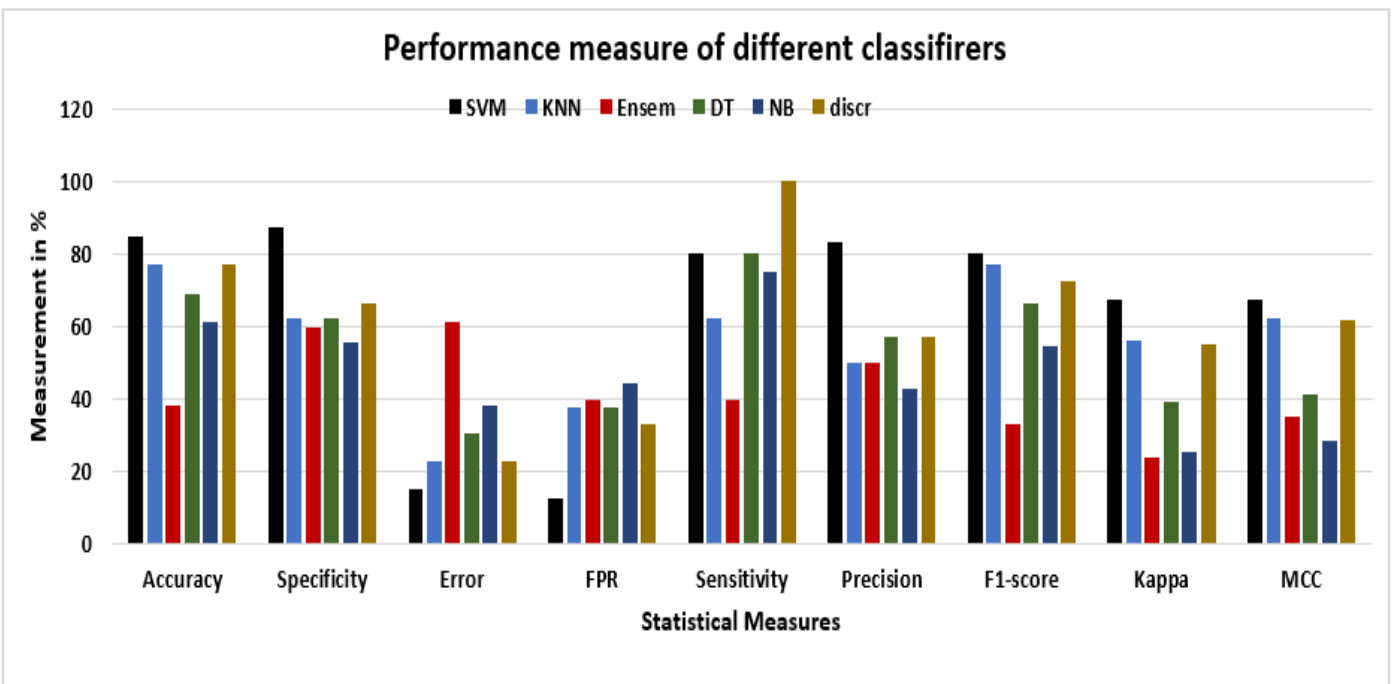

Figure 9 Overall performance of all classifiers

\section{Discussion}

From the results, we can observe that SVM has improved accuracy of $55.59 \%, 28.95 \%, 20.07 \%$, $28.95 \%$, and $11.19 \%$ compare to $\mathrm{KNN}$, ensemble, DT, NB, and discriminant classifiers. SVM has reduced error of $75 \%, 60 \%, 50 \%, 60 \%$, and $33.31 \%$ compare to KNN, ensemble, DT, NB, and discriminant classifiers. SVM gives improved sensitivity of $75 \%, 25 \%, 0 \%, 6.25 \%$, and $-25 \%$, improved specificity of $8.57 \%, 57.14 \%, 28.57 \%$, $36.5 \%$, and $23.8 \%$, improved precision of $40 \%$, $55 \%, 31.43 \%, 48.57 \%$, and $31.43 \%$, FPR reduced by $37.5 \%, 80 \%, 66.67 \%, 71.87 \%$, and $62.49 \%$, F1-score improved by $75 \%, 31.88 \%, 16.67 \%, 31.81 \%$, and $9.1 \%$, improved MCC of $55.56 \%, 35.85 \%, 38.55 \%$,
$58 \%$, and $8.56 \%$, improved kappa of $65.8 \%, 53.22 \%$, $41.43 \%, 62.53 \%$, and $18.27 \%$ compare to other classifiers labelled as KNN, ensemble, DT, NB, and discriminant classifiers. Discrimint analysis classifier gives highest sensitivity of $100 \%$. From the observation, we can say that SVM classifier gives improved performance compare to other classifiers. The shape of liver and location of tumor changes from image to image. Hence the features extracted from the CT liver image has irreguraly distributed features. Since SVM is able to perform well for irreguraly distributed features whereas KNN performance reduces due to irregular features and NB assumes all features are independent. So, SVM 
classifier is able to give better performance compare to other classifiers.

The accuracy of SVM classifier for the dataset considered is only $84.6 \%$. It can be increased further by considering larger dataset. In this paper, we have used region growing method for liver segmentation. In this method, the seed point should be selected manually to extract the liver region. Instead of using manual segmentation of liver, automatic segmentation of liver is possible with neural network $(\mathrm{NN})$. The NN models can be used for both segmentation and classification. But to train the NN model, large dataset should be used, otherwise the performance of NN will not be good. A complete list of abbreviations is shown in Appendix I.

\subsection{Limitation}

In this paper, the dataset consist of $68 \mathrm{CT}$ images with 86 features. Since the dataset is small, the optimization algorithms are not used to train the model. If the dataset is increased then to reduce the training time and testing time optimization algorithms must be used. By using optimization algorithms, classifier can be trained with selected features most suitable for analysis instead of using all features. Further by using large dataset, the performance of classifier can be improved.

\section{Conclusion}

Liver tumor classification is difficult task, because the shape of the liver, tumor and lesion texture changes with liver abnormalities. Hence, liver tumor classification is current research problem. In paper, we have used different classification methods for classifying liver abnormalities as LA, and HCC. Here we have used $64 \mathrm{CT}$ images, in which 51 images are used for training and remaining images for testing. SVM classifier has given highest accuracy, sensitivity, specificity, precision, F1-score, and MCC, and lowest error, and FPR compared to other classifiers. In future, we are going to study effect of heuristic-based optimization algorithms in classification. Deep learning algorithms with heuristic-based optimization techniques, and large data set can give best classification performance. Future work includes collecting large data set of at least $250 \mathrm{CT}$ images, extraction of liver and tumor region from CT images automatically. Optimization algorithms will be used in machine learning algorithms to improve the performance of classifier and to reduce the computation time.
Acknowledgment

None.

Conflicts of interest

The authors have no conflicts of interest to declare.

Authors contribution statement

Munipraveena Rela: Data collection, conceptualization, investigation, writing - original draft, analysis and interpretation of results. Suryakari Nagaraja Rao: Conceptualization, supervision, writing - review and editing. Patil Ramana Reddy: Conceptualization, supervision, writing - review and editing.

\section{References}

[1] Rela M, Suryakari NR, Reddy PR. Liver tumor segmentation and classification: a systematic review. In IEEE-HYDCON 2020 (pp. 1-6). IEEE.

[2] Jemal A, Bray F, Center MM, Ferlay J, Ward E, Forman D. Global cancer statistics. CA: A Cancer Journal for Clinicians. 2011; 61(2):69-90.

[3] Bucak İÖ, Baki S. Diagnosis of liver disease by using CMAC neural network approach. Expert Systems with Applications. 2010; 37(9):6157-64.

[4] Ruskó L, Bekes G, Fidrich M. Automatic segmentation of the liver from multi-and single-phase contrast-enhanced CT images. Medical Image Analysis. 2009; 13(6):871-82.

[5] Das A, Das P, Panda SS, Sabut S. Detection of liver cancer using modified fuzzy clustering and decision tree classifier in CT images. Pattern Recognition and Image Analysis. 2019; 29(2):201-11.

[6] https://www.ncbi.nlm.nih.gov/books/NBK538230/. Accessed 23 July 2021.

[7] Balogh J, Victor III D, Asham EH, Burroughs SG, Boktour M, Saharia A, et al. Hepatocellular carcinoma: a review. Journal of Hepatocellular Carcinoma. 2016; 3:41-53.

[8] Li BN, Chui CK, Chang S, Ong SH. A new unified level set method for semi-automatic liver tumor segmentation on contrast-enhanced CT images. Expert Systems with Applications. 2012; 39(10):9661-8.

[9] Rela M, Rao SN, Reddy PR. Liver tumor segmentation using superpixel based fast fuzzy C means clustering. International Journal of Advanced Computer Science and Applications. 2020; 11(11):380-7.

[10] Ranjbarzadeh R, Saadi SB. Automated liver and tumor segmentation based on concave and convex points using fuzzy c-means and mean shift clustering. Measurement. 2020.

[11] Machhale K, Nandpuru HB, Kapur V, Kosta L. MRI brain cancer classification using hybrid classifier (SVM-KNN). In international conference on industrial instrumentation and control 2015 (pp. 60-5). IEEE.

[12] Nadira T, Rustam Z. Classification of cancer data using support vector machines with features selection method based on global artificial bee colony. In AIP conference proceedings 2018. AIP Publishing LLC. 
[13] Starmans MP, Miclea RL, Van DVSR, Niessen WJ, Thomeer MG, Klein S. Classification of malignant and benign liver tumors using a radiomics approach. In medical imaging: image processing 2018. International Society for Optics and Photonics.

[14] Zhen SH, Cheng M, Tao YB, Wang YF, Juengpanich $\mathrm{S}$, Jiang ZY, et al. Deep learning for accurate diagnosis of liver tumor based on magnetic resonance imaging and clinical data. Frontiers in Oncology. 2020.

[15] Trivizakis E, Manikis GC, Nikiforaki K, Drevelegas $\mathrm{K}$, Constantinides M, Drevelegas A, et al. Extending 2-D convolutional neural networks to 3-D for advancing deep learning cancer classification with application to MRI liver tumor differentiation. IEEE Journal of Biomedical and Health Informatics. 2018; 23(3):923-30.

[16] Kutlu H, Avcı E. A novel method for classifying liver and brain tumors using convolutional neural networks, discrete wavelet transform and long short-term memory networks. Sensors. 2019; 19(9):1-16.

[17] Rela M, Nagaraja RS, Ramana RP. Optimized segmentation and classification for liver tumor segmentation and classification using oppositionbased spotted hyena optimization. International Journal of Imaging Systems and Technology. 2021; 31(2):627-56.

[18] Balagourouchetty L, Pragatheeswaran JK, Pottakkat B, Ramkumar G. GoogLeNet-based ensemble FCNet classifier for focal liver lesion diagnosis. IEEE Journal of Biomedical and Health Informatics. 2019; 24(6):1686-94.

[19] Budak Ü, Guo Y, Tanyildizi E, Şengür A. Cascaded deep convolutional encoder-decoder neural networks for efficient liver tumor segmentation. Medical Hypotheses. 2020.

[20] Zhang D, Chen B, Chong J, Li S. Weakly-supervised teacher-student network for liver tumor segmentation from non-enhanced images. Medical Image Analysis. 2021.

[21] Devi RM, Seenivasagam V. Automatic segmentation and classification of liver tumor from CT image using feature difference and SVM based classifier-soft computing technique. Soft Computing. 2020; 24(24):18591-8.

[22] Krishan A, Mittal D. Ensembled liver cancer detection and classification using CT images. Proceedings of the Institution of Mechanical Engineers, Part H: Journal of Engineering in Medicine. 2021; 235(2):232-44.
[23] Burges CJ. A tutorial on support vector machines for pattern recognition. Data Mining and Knowledge Discovery. 1998; 2(2):121-67.

[24] Keller JM, Gray MR, Givens JA. A fuzzy k-nearest neighbor algorithm. IEEE Transactions on Systems, Man, and Cybernetics. 1985; 15(4):580-5.

[25] https://analyticsindiamag.com/basics-of-ensemblelearning-in-classification-techniques-explained. Accessed 23 August 2021.

[26] Sairamya NJ, Susmitha L, George ST, Subathra MS. Hybrid approach for classification of electroencephalographic signals using time-frequency images with wavelets and texture features. In intelligent data analysis for biomedical applications 2019 (pp. 253-73). Academic Press.

[27] Chandra PK, Bharathi C. Texture analysis using GLCM \& GLRLM feature extraction methods. International Journal for Research in Applied Science \& Engineering Technology. 2019; 7(5):2059-64.

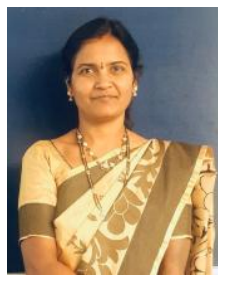

Munipraveena Rela received B.Tech from Jawaharlal Nehru Technological university in 2001, M.Tech from visveswaraiah Technological university in 2005. Currently pursing $\mathrm{PhD}$ under JNTUA, Ananthapur, Andhra Pradesh, India. She has 15 years of teaching experience. Currently working as Associate Professor in CMR Institute of Technology, Hyderabad. She has published more than 20 research papers in various journals. She is life member of ISTE.

Email: chagpraveena@gmail.com

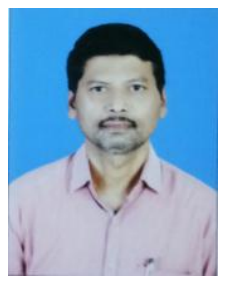

Dr. Suryakari Nagaraja Rao received M. Tech in Digital Systems and Computer Electronics from J.N.T.University, Hyderabad in 1998 and Ph.D in the area of Signal and Image Processing from Jawaharlal Nehru Technological University Ananthapuramu (JNTUA) in 2011. Presently he is working as Professor \& Head, Department of ECE, G. Pulla Reddy Engineering college(Autonomous), Kurnool, AP, India. He has 28 years of experience from this 7years in the Industry and 21 Years in Teaching. His interests include signal processing, Image Processing and VLSI. He has more than 50 publications in various International/National Journals/Conferences. $\mathrm{He}$ is currently guiding $7 \mathrm{PhD}$ scholars. He is Life member of ISTE, Life member of Instrument Society of India (ISOI),member of IEEE.

Email: Suryakari2k9@gmail.com 


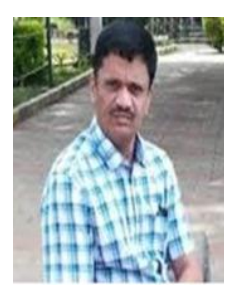

Dr. Patil Ramana Reddy received the M.E (Electronics) from SGGSCE\&T, Nanded, Maharashtra in 1994 and Ph.D in the area of Image Processing from Jawaharlal Nehru Technological University Ananthapuramu (JNTUA) in 2010. Presently he is working as Professor \& Head, Department of ECE, JNTUA College of Engineering, Ananthapuramu, AP, India. He has 25 years of teaching \& 17 years of research experience. His interests include signal processing and Image Processing. He has about 99 publications in various International/National Journals/Conferences. $\mathrm{He}$ is currently guiding 15 and guided $8 \mathrm{PhD}$ students. $\mathrm{He}$ is fellow of IEI and IETE and life member of ISTE.

Email: prrjntu@gmail.com

\begin{tabular}{lll}
\multicolumn{2}{l}{ Appendix I } \\
\hline S. No. & Abbreviation & Description \\
\hline 1 & 2D & Two Dimensional \\
\hline 2 & 3D & Three Dimensional \\
\hline 3 & CAD & Computer-Aided Design \\
\hline 4 & CNN & Convolution Neural Network \\
\hline 5 & CT & Computed Tomography \\
\hline 6 & DT & Decision Tree \\
\hline 7 & DWT & Discrete Wavelet Transform \\
\hline 8 & FCNet & Fully Connected Neural Network \\
\hline 9 & FPR & False Positive Rate \\
\hline 10 & GABC & Global Artificial Bee Colony \\
\hline 11 & GLCM & Gray-Level Co-Occurrence Matrix \\
\hline 12 & HCC & Hepatocellular Carcinoma \\
\hline 13 & KNN & K-Nearest Neighbor \\
\hline 14 & LA & Liver Abscess \\
\hline 16 & LBP & Local Binary Pattern \\
\hline 17 & LSTM & Long Short-Term Memory \\
\hline 18 & MCC & Matthews Correlation Coefficient \\
\hline 19 & MR & Magnetic Resonance \\
\hline 20 & NB & Magnetic Resonance Imaging \\
\hline 21 & NN & Naive Bayes \\
\hline 22 & ReLU & Neural Network \\
\hline 23 & RMS & Rectified Linear Unit \\
\hline 24 & RNN & Reot Mean Square \\
\hline 25 & SVM & Support Vector Machine \\
\hline & & \\
\hline
\end{tabular}

